\title{
Trauma-informed hospital security: a policy for intimate partner violence in a pediatric healthcare setting
}

\author{
Allison V. Bautista ${ }^{1}$, Katherine C. Obenschain ${ }^{2,3}$, Joseph T. Potterton $^{4}$, Ashlee L. Murray ${ }^{2,5,6}$ \\ ${ }^{1}$ Master of Public Health Program, University of Pennsylvania, Philadelphia, PA, USA; ${ }^{2}$ Center for Violence Prevention, Children's Hospital \\ of Philadelphia, Philadelphia, PA, USA; ${ }^{3}$ Bilingual Domestic Violence Program, Lutheran Settlement House, Philadelphia, PA, USA; ${ }^{4}$ Security \\ Department, Children's Hospital of Philadelphia, Philadelphia, PA, USA; ${ }^{5}$ Division of Emergency Medicine, Children's Hospital of Philadelphia, \\ Philadelphia, PA, USA; ${ }^{\circ}$ Department of Pediatrics, University of Pennsylvania School of Medicine, Philadelphia, PA, USA \\ Contributions: (I) Conception and design: All authors; (II) Administrative support: AL Murray, KC Obenschain, JT Potterton; (III) Provision of \\ study materials or patients: AL Murray, KC Obenschain, JT Potterton; (IV) Collection and assembly of data: All authors; (V) Data analysis and \\ interpretation: AV Bautista; (VI) Manuscript writing: All authors; (VII) Final approval of manuscript: All authors. \\ Correspondence to: Ashlee L. Murray. Center for Violence Prevention, Children's Hospital of Philadelphia, Philadelphia, PA 19104, USA; Division \\ of Emergency Medicine, Children's Hospital of Philadelphia, Philadelphia, PA 19104, USA; Department of Pediatrics, University of Pennsylvania \\ School of Medicine, Philadelphia, PA 19104, USA. Email: murraya2@email.chop.edu.
}

Background: Increasing numbers of providers are adopting "trauma-informed approaches" in patient care models to address intimate partner violence (IPV), but most hospitals' security policies and practices do not incorporate trauma-informed approaches. Pre-existing security culture and differing approaches to trauma-informed care necessitate policy-level interventions. To support the physical and emotional safety of IPV survivors within the hospital environment, the security leaders at our tertiary-care, inner-city pediatric hospital expressed the need for a trauma-informed IPV security policy.

Methods: Policy development was conducted by our workgroup consisting of the Security Operations Manager, Director of our hospital's IPV task force who is also a Pediatric Emergency Medicine physician, IPV advocates from our community partnership agency, our hospital's IPV specialist, a trauma-informed expert, a social worker, and a Master of Public Health graduate student. Biweekly video conference meetings were held to iteratively and collaboratively refine elements throughout policy development.

Results: The policy encompassed four sections: (I) how to ensure safety for all individuals affected by IPV, (II) how to refer individuals impacted by IPV to resources, (III) maintaining confidentiality, and (IV) reporting to local law enforcement. Two job aids were also created entitled "implementing a traumainformed approach" and "de-escalation techniques".

Conclusions: Creating a trauma-informed, IPV security policy is integral to bridging gaps between hospital security and healthcare providers, and providing a framework for accountability and standard of care. Engagement from security leaders is necessary for changing perceptions and attitudes towards traumainformed approaches and creating a culture more supportive for survivors of IPV.

Keywords: Intimate partner violence (IPV); trauma-informed; hospital security; pediatric

Received: 07 December 2020; Accepted: 02 April 2021; Published: 25 December 2021.

doi: $10.21037 /$ jhmhp-20-152

View this article at: http://dx.doi.org/10.21037/jhmhp-20-152

^ ORCID: Allison V. Bautista, 0000-0002-5794-7890; Ashlee L. Murray, 0000-0002-4881-5626. 


\section{Introduction}

According to a recent poll from June 2020, about one-third of Americans distrust local police forces (1). This distrust is echoed in the underreporting of intimate partner violence (IPV) incidents $(2,3)$ - especially during the COVID-19 pandemic when IPV prevalence increased (4,5) — and further reinforced by evidence detailing the high frequency of police mishandling sexual assault cases (6). It is becoming increasingly evident that police officers must adopt a more empathetic, mindful approach to prevent needless harm and traumatization towards IPV survivors (7). Within this sector are hospital security officers who hold a particularly important role, as hospitals are often the first touchpoint in addressing IPV (8). Since approximately $26 \%$ of children in the United States have been exposed to adult IPV (9), increasing numbers of pediatric health professionals are adopting "trauma-informed approaches" in patient care models $(10,11)$, which posit the idea that anyone may have a history of trauma (12). Interventions such as screening and referral to resources are common in addressing IPV (13), but these efforts struggle to integrate hospital security officers, which can introduce vulnerabilities in care (14).

Most hospitals' security practices and policies do not incorporate trauma-informed approaches, which may be attributed to the siloed nature in which security departments operate within hospital settings (14). Common methods for addressing workplace violence, crisis intervention, and conflict management include restraints use, seclusion, and involuntary medication (15), but these methods can trigger traumatic memories in individuals with histories of physical and/or sexual abuse (16,17). Security personnel have expressed frustration about the lack of security-clinician communication and what they perceive to be hospital staff's surface-level understanding of security roles and responsibilities (14). Despite this tension, there is growing interest among hospital security leaders on how to implement trauma-informed approaches (14). Individual-level strategies to address these concerns include security officers shadowing clinicians, which may foster better working relationships and greater understanding of the patient experience (14). For example, at one New England hospital, security officers shadowed nurses, therapists, and social workers, which resulted in greater communication on how to best support each other during patient incidents (14). However, this type of intervention relies on the current security culture and individual attitudes and perceptions, which may create inconsistencies in the quality of care. Therefore, to support the physical and emotional safety of IPV survivors, the security leaders at our tertiary-care, inner-city pediatric hospital expressed the need for a trauma-informed IPV security policy.

Many existing IPV policies within pediatric healthcare settings do not explicitly define the security department's role, as they mostly apply to healthcare providers, and language pertaining to security is often vague or nonexistent $(18,19)$. Our hospital had two policies that demonstrated this gap. The first was our hospital's policy entitled "Policy: Intimate Partner Violence", in which security was not mentioned (20). The second was our hospital's "Violence in the Workplace" policy, which instructs employees to report any concerns of violence to the security department to "take appropriate action to investigate and mitigate the threat" (21). At the time of policy development, there was a dearth of pediatric hospital policies to guide the security department in responding to IPV, and no such policy at our hospital. The primary aim of this project was to develop a trauma-informed policy for our hospital that guides the security department on how to appropriately respond to families and employees experiencing IPV, specifically incidents of IPV that occur at the hospital itself. In doing so, we can also inform future healthcare institutions about how to develop traumainformed security policies and better integrate security's role into the healthcare team.

\section{Methods}

The need for a trauma-informed IPV policy for our hospital's security department was identified in 2018 by our hospital-wide IPV task force and the Security Operations Manager. Our policy was created starting in January 2020 and completed in July 2020.

\section{Phase 1. Identify the workgroup}

The Security Operations Manager was identified as the main contact to obtain security input for policy development, since this individual could provide insight into existing security protocols and how the policy can support security's workflow. Additional members of the workgroup included the Director of our hospital's IPV task force, who is also a Pediatric Emergency Medicine physician with knowledge about hospital culture and how issues of IPV are typically handled; IPV advocates from our community partnership agency with expertise 
in responding to IPV in healthcare; our hospital's IPV specialist who works closely with individuals experiencing IPV; a social worker, since they are most directly involved with responding to the needs of individuals experiencing IPV; an expert in trauma-informed care, who would help identify the elements of an effective trauma-informed policy; and a Master of Public Health graduate student, who was responsible for writing the policy in collaboration with the workgroup.

\section{Phase 2. Review existing policies}

Further research was conducted on existing IPV policies that outlined security's roles and responsibilities. Our hospital's policy database was searched for any violencerelated policies, including IPV and workplace violence, in order to determine current security responses to IPV. In addition, phone interviews with representatives at two other pediatric hospitals across the nation were conducted to inform policy development. These hospitals were selected using a search engine to identify top-ranking pediatric hospitals in the United States, and a subsequent search was conducted to find the contact information of individuals associated with IPV policies and procedures at each hospital. The physician in our workgroup also had contact information for IPV leaders at other pediatric hospitals.

Out of seven pediatric hospitals that were contacted, only two responded and agreed to a phone interview. The first phone interview was conducted with the Senior Community Initiatives Coordinator at a southern pediatric hospital who conducted a needs assessment for IPV screening at their hospital. This hospital did not have an IPV policy for security but did have a workflow for IPV screening in their prenatal and maternity care center where security officers played a major role in patient intake. Since patients and their partners often arrived at the health center together, security checked in patients separately so that the nurse could bring them back for IPV screening before their partner joins them. The second phone interview was conducted with a physician who served as the Co-Director of Research in the Division of Emergency Medicine at a midwestern pediatric hospital. This individual informed us that their hospital also did not have an IPV policy for security, but that this need has been raised in their hospital.

Copies of violence-related policies from two pediatric hospitals that were not interviewed were also obtained via email. One policy was obtained from a pediatric hospital located in the same city as our hospital, and the other policy came from a southeastern pediatric hospital. These policies also did not mention security. Lastly, a PubMed search was conducted using search terms "intimate partner violence", "security", "pediatric", "policy", "trauma-informed", "domestic violence", and "safety" to identify articles that would inform policy development.

\section{Phase 3. Schedule policy development meetings with stakeholders}

Biweekly meetings were held with the workgroup to collectively brainstorm ideas for the policy. Initial workgroup meetings were held without security for two reasons: (I) to review evidence and existing policies, and prepare a draft policy outline for the first meeting with security; and (II) it was difficult to identify the proper security contact and engage the security department to schedule an initial meeting. The Security Operations Manager assumed responsibility for policy development after our initial contacts did not respond.

During these preliminary meetings, an outline for the policy was created. The workgroup decided that three policies were needed because we anticipated that security responses would vary depending on the population experiencing IPV: employees, families and caregivers, and patients. During the first meeting with the Security Operations Manager, the outline was presented. Subsequent biweekly video conference meetings with the Security Operations Manager were held over 3 months. It was eventually decided that only one overarching policy was necessary, as security officers have the same response to IPV regardless of population, and rarely respond to incidents of teen dating violence or IPV among patients. A finalized version of the policy outline was completed. It was also decided that we would create two job aids, which are one-to-two-page documents that provide information to support how security carries out their responsibilities. For example, the job aid "De-Escalation Techniques" outlines concepts of de-escalation and walks through the steps of conflict management. The job aids were deemed necessary because security officers may hold varying levels of understanding of trauma-informed approaches, and a readily available document would promote consistency in their responses. The policy and job aids were revised and sent via email to the workgroup between video conference meetings so that feedback could be iteratively discussed at 
each meeting.

\section{Phase 4. Submit for hospital approval}

The policy and corresponding job aids were submitted to the Environment of Care Committee within our hospital for review on July $8^{\text {th }}, 2020$ as part of the hospital's Security Manual. Response from the committee and approval occurred within 1 month. At the time this manuscript was written, the policy was approved and published as a standard of care at our institution.

\section{Statistical analysis}

Policy development did not include quantitative measurement; therefore, the authors did not conduct any statistical analyses.

\section{Results}

The purpose of our policy was "to outline the steps for when the security department responds to incidents in which individuals at (our hospital) are experiencing IPV. Appropriate response by security officers will include: ensuring safety for all individuals involved, referrals to appropriate resources, maintaining confidentiality, (and) reporting to local law enforcement" (https://cdn. amegroups.cn/static/public/jhmhp-20-152-1.docx). We also included a list of terms and definitions that were essential to understanding the policy.

\section{Definitions}

Our policy differentiated between the terms "Family Violence" and "Intimate Partner Violence". While there is overlap between these terms, "Family Violence" was defined as any act of violence between family or household members (22), which includes child abuse and elder abuse, while "Intimate Partner Violence" was defined as a range of behaviors perpetrated by an intimate or a dating partner, with the intent to gain or maintain power and control over their partner (23). Since the "Power and Control Wheel" is a notable concept in IPV literature (24), we also felt it was necessary to define "Power and Control" as the pattern of abuse and violence used to establish and maintain control over an intimate partner, but is not limited to physical or sexual violence and could include economic abuse, isolation, coercion, and threats (24). Our policy also defined "Mandated Reporter", which refers to an individual who is required to report suspected or confirmed child abuse (25), and we provided a link to the statewide online portal for filing reports.

\section{Policy section 1. Ensuring safety}

Our review of current security training materials revealed a focus on verbal and non-verbal de-escalation techniques, proxemics, and strategies to promote personal safety, such as physical defensive tactics (26-28). For example, security officers were instructed to anticipate potential violence and be mentally prepared to act if it arises: "Theft, violence and harassment are everywhere. Be smart, don't think it won't happen to you, be prepared mentally and physically" (26). From these materials, it was also apparent that security officers, although not often necessarily trained in the traumainformed discipline, actually already employ many traumainformed approaches in their practice: "Empathy, if done correctly, will begin the process of de-escalation immediately... whether you can or can't provide a solution, the key is to make some type of physical effort showing them you tried" (28).

Using this information, we determined that the policy should instruct security officers to immediately notify their shift supervisor and the manager of the unit/clinic during an incident of IPV, as this would initiate a response for additional support if needed. As a precautionary measure, the policy also instructs the security officer to separate the parties involved and hold one-on-one conversations with the victim, alleged perpetrator of IPV, and any other individual involved. This would prevent further violence between these two parties and allow the security officer to gather a more comprehensive account of the event; however, security officers can only enforce Protection from Abuse (PfA) orders if the individual has a physical copy with them, as per Pennsylvania state law (29). After security officers de-escalate the situation, they are to refer to a social worker who would address the specific needs of the person experiencing IPV.

The initial de-escalation response through communication was identified as a key component of a trauma-informed approach, since it prevents the need for using physical tactics that could potentially be traumatizing. To help build on security's understanding of trauma-informed approaches, the job aid entitled "implementing a traumainformed approach" was created, which outlined the four steps of trauma-informed care and key principles to promote empathy and respect (https://cdn.amegroups.cn/ static/public/jhmhp-20-152-2.docx). Our team also created 
the job aid entitled "de-escalation techniques" (https://cdn. amegroups.cn/static/public/jhmhp-20-152-3.docx) using security training materials, as there was no formal document that outlined these techniques that are taught to all security officers at our hospital. While these job aids were intended to supplement our security policy, we also felt that they may be useful resources for other departments such as patient care.

Our team also believed that our policy should emphasize how child abuse may be co-occurring with IPV (30). As part of a trauma-informed approach, security officers should not discuss IPV in front of children age 2 or older because some children repress their experiences with IPV and discussing it with them may be retraumatizing (31). Our policy also describes the parameters and implications of involving Child Protective Services (CPS), as inappropriate initiation of CPS may escalate danger and risk of harm and trauma among children (32). Additional links and resources included information on mandatory reporting for child abuse, policies on visitation guidelines and visitor restrictions, policies for employees experiencing workplace violence, and referral to our on-site IPV community advocate.

\section{Policy section 2. Referral to resources}

During our meetings with the Security Operations Manager, a common theme mentioned was security officers' hesitation to acknowledge the personal impact of their job and discuss how a distressing event has affected them. They often downplay the magnitude of their distress and will not initiate debriefings with the security team. Debriefings are largely dependent on the shift supervisor who would assess the need for one, but this introduces variation in supporting security officers; while one supervisor may more readily debrief with the officers on shift, another supervisor may not see the need for debriefings altogether. Another theme that emerged was how security officers often feel frustrated when individuals experiencing IPV refuse resources that could potentially help them.

We felt that security officers should have a basic understanding of appropriate resources so that they can activate those channels when requested, which prompted the inclusion of links within our policy to child referral services, contact information of the IPV specialist, and how to access the local and national domestic violence hotlines. It was especially important that our policy emphasized how some individuals may not want resources, since a trauma-informed approach respects the choices made by an individual who has experienced IPV (12): "If the survivor of IPV expresses that they do not need help, it is a trauma-informed response to respect their wishes. Unwanted intervention may put the individual(s) and their family more at risk and can perpetuate the "power and control" cycle" (https://cdn.amegroups.cn/ static/public/jhmhp-20-152-1.docx). We acknowledged that the resulting frustration felt by security officers originates from well-intentioned motivation to prevent further violence and harm, which is why our policy also included information on secondary traumatic stress, defined as "the emotional duress that results when an individual hears about the firsthand trauma experiences of another" (33). However, to ensure that the policy is supportive of current security practices, it was necessary to acknowledge the hierarchy related to debriefings: "The responding security officer may consult with the IPV specialist on how to appropriately respond... The security supervisor on duty may debrief with the security officers and staff members who responded to the incident to better inform future response, safety planning, and initiate mental bealth assistance. The IPV specialist's presence can be requested for these debriefings" (https://cdn.amegroups.cn/static/public/ jhmhp-20-152-1.docx). This language respects the security culture, while also informing security officers about the resources available for them.

\section{Policy section 3. Maintaining confidentiality}

Main themes regarding confidentiality that arose during our meetings with security were the need for proper documentation and debriefing. Confidentiality must be maintained to protect individuals experiencing IPV and prevent jeopardizing their safety (34)—for example, keeping information on an individual's whereabouts away from perpetrators of violence.

In our policy, it was first important to differentiate between "confidentiality" and "privacy" because these two terms are often interchanged. Confidentiality refers to the duty of an individual entrusted with health information to keep that information private, while privacy refers to an individual's right to keep their health information private (35). A trauma-informed approach supports the notion that individuals should share only what they feel comfortable with, but also acknowledges that certain pieces of information are necessary to address their needs $(36,37)$. Our policy instructed security officers to refrain from sharing information with individuals who were not directly involved in or responding to the incident; however, 
we also referenced information on debriefings and contact information of the IPV specialist, which are approved ways that security officers may discuss the personal impact of their job. Maintaining confidentiality also implies that any physical notes taken during the incident are shredded in the HIPAA-compliant bin. After the initial response, security officers must document in the online Incident Reporting form, as all the information stored in this database is kept confidential. This form includes fields for the name of the person reporting, type of incident (e.g., alarm, damage, fire, injury, or theft), synopsis/overview, incident location, and date and time that the incident occurred. We also provided a screenshot of the Incident Reporting user view within the policy so that security officers can confirm if they are documenting appropriately.

\section{Policy section 4. Reporting to local law enforcement}

In the state where our hospital is located, incidents of IPV do not have to be reported unless a weapon is involved (38). The Security Operations Manager identified three main instances when the security department involves local law enforcement: (I) when the alleged perpetrator of IPV poses an immediate danger to an individual, (II) when a deadly weapon is used, or (III) to initiate a chain of custody for physical violence.

In our policy, we outlined these three instances and provided a link for information about mandatory reporting in the healthcare environment. Most IPV incidents that occur in our hospital do not fit the criteria for involving local law enforcement; therefore, we stated that security officers may consult with the IPV specialist for advice on how to appropriately respond. We also acknowledged that since these events do not occur frequently, they may be particularly distressing when they do happen. Within this section of our policy, we felt it was important to reiterate that the security supervisor may hold debriefings and included the link for the Employee Assistance Program, which supports the personal and mental health needs of employees.

\section{Discussion}

\section{Organizational implications}

Evaluation of the policy is important in measuring the policy's success and can be conducted using the "Reach", "Effectiveness", "Adoption", "Implementation", and
"Maintenance" framework, or the RE-AIM framework (39). Since this policy was only recently developed, we have not had the opportunity to evaluate it to-date but plan to do so according to this framework. We believe this can also serve as a model for other hospitals seeking to develop, expand, or adjust their own policies.

"Reach" refers to the number of security officers who understand and are aware of the policy (39). It can be improved by holding mandatory in-service security staff meetings to present the policy. These meetings can also serve to build understanding of trauma-informed care and its importance, hold open discussions, and answer any questions that security staff may have.

"Effectiveness" refers to the impact of the policy on our intended outcomes (39), which are disseminating knowledge about trauma-informed care among security staff, enhancing our hospital-wide culture of safety, and improving the experiences of IPV survivors who are visiting or employed by our hospital. Effectiveness can be measured through surveys regarding security staff's beliefs and attitudes towards trauma-informed care and if they believe our hospital is actively working towards creating a safe environment. Individuals who have experienced IPV can also be interviewed regarding their experiences with security.

Measuring "Adoption" and "Implementation" may occur together. "Adoption" refers to the number of security staff who have actually implemented the policy guidelines within their practice, while "Implementation" refers to how closely practice aligns with the policy, also known as fidelity (39). These factors may be measured through direct observation of security's workflow when responding to IPV incidents. Focus groups with security staff can also be conducted to gather positive and negative feedback about the policy. For example, security staff can voice what they find helpful in the policy versus barriers to implementation and discuss suggestions for improvement. Objective metrics of implementation may also include staff productivity, hospital cost and revenue, the number of staff sick days, and uptake of staff services, such as the Employee Assistance Program.

"Maintenance" can be measured at both the individual and institutional level. It refers to the long-term sustainability of the policy, such as if the policy has been integrated into the security officer's routine and normalized as part of the institution's culture (39). Evaluating maintenance may occur on a quarterly or biannual basis for the first year and annual basis for subsequent years. The same surveys used for measuring effectiveness, adoption, 
and implementation may be used to determine whether certain variables increased or decreased over time, such as debriefings among security staff after IPV incidents.

\section{Implementation barriers and potential solutions}

We identified potential barriers to implementation and offer some suggestions for healthcare institutions seeking to implement their own trauma-informed IPV security policies. Fidelity of implementation often requires transforming the attitudes of healthcare professionals as well as changing the structural environment (40). Categories of implementation barriers therefore include (I) personal factors, such as security officers' lack of familiarity or self-efficacy with trauma-informed responses to IPV; (II) external factors, which include organizational and social norms; and (III) guideline-related factors, which consider how our policy is an early contribution to the evidence-base of trauma-informed hospital security policies for IPV (40).

Our policy describes the collaboration between security officers and multiple departments when responding to IPV incidents, such as social workers, the shift supervisor, or the unit/clinic manager. However, potential obstacles may prevent communication between security and other disciplines; for example, social workers may not be readily available, or an overwhelming workload may discourage security officers from notifying the shift supervisor or unit manager. Potential solutions include notification of the patient's medical care team with the expectation that the nurse or physician on shift would schedule a timely consultation with a social worker. Notification of IPV incidents may also be incorporated into daily huddle, so that security officers can inform multiple hospital actors at once. It may be worthwhile to also advocate for a full-time or oncall social worker to increase access to these services.

Another major concern regarding policy implementation is security officers potentially discounting IPV and traumaspecific resources, which may relate to the prevailing organizational or security culture that downplays the personal impact of one's job. Healthcare institutions may address this issue by utilizing IPV champions to help facilitate culture change within their organization and normalize the uptake of trauma-informed resources. Additionally, hospitals may create a standardized process that activates when security officers express IPV-related concerns like secondary traumatic stress; this process should go beyond decreasing work hours and seek to comprehensively address the needs of staff.
Additional barriers to implementation include obstacles in de-escalating and reporting IPV incidents. Security officers may lack the physical space to separate the parties involved in an IPV incident, due to reasons like hospitals being overwhelmed from an influx of COVID-19 patients. Further, variations in laws, local resources, and regional culture may complicate how security officers obtain back-up during unfolding IPV incidents or refer to law enforcement. For example, law enforcement may respond differently to IPV, and security officers may not receive the support they expected. Role-play training may help security officers anticipate how various situations may unfold and identify factors that are unique to the organization and surrounding community. Healthcare institutions may also find it necessary to identify IPV community resources and advocates that can inform security officers' responses to IPV. These resources may be compiled in an easily accessible document such as a job aid so that security officers can feel more supported on the job.

\section{Limitations of project}

Participation from security was identified as a major hurdle during policy development, as security was initially not responsive to our emails, and it was difficult setting up an initial meeting with them. This factor points to concerns over security engagement; while the Security Operations Manager and security leaders may hold great interest for trauma-informed IPV approaches, the rest of the security department may not. Our workgroup would have liked to also collaborate with security officers who did not hold leadership positions in order to gauge current perceptions and attitudes of the workforce, since they may differ from that of security leaders. Prior to policy development, there was also no formal baseline measurement of security staff's existing knowledge and attitudes on trauma-informed care, IPV, security culture, and working relationships with other departments, such as clinicians. It would have been helpful to see if these variables changed after policy implementation in order to improve the policy. Since we were not able to collaborate with more members of the security team, we are unsure about how the new policy will translate into practice, or how closely practices will align with what is outlined in the policy. If the policy is not implemented as intended, it may lose its effectiveness in fostering a trauma-informed culture hospital-wide.

The COVID-19 pandemic and quarantine also occurred during policy development. Other issues may have taken 
greater precedence than our policy, and stakeholder engagement may have been lower than anticipated. Our communication had to occur virtually, such as through video conferencing, which introduced additional learning curves.

Our policy was also created without guidance from an existing template, since no IPV policies related to traumainformed security approaches were found. There is uncertainty surrounding effectiveness and necessity of some items in the policy, and we may have excluded important factors that are not readily apparent without testing how the policy works in practice. New best practices may emerge as more hospital security departments adopt trauma-informed approaches.

\section{Strengths of project and implications for other hospitals}

A major strength of our policy development was collaboration with the Security Operations Manager, who is the Head of Security at our hospital. Building a traumainformed approach among all members of the security department requires engagement from security leaders who act as change agents within an organization and can influence security culture, perspectives, and attitudes. The Security Operations Manager also provided insight into the day-to-day operations of the security department, which helped tailor our policy to the specific needs of security's workflow and address gaps in practice.

Our policy workgroup also consisted of individuals from various disciplines who hold different approaches towards addressing IPV. This aspect fostered an exchange of ideas and insight into other perspectives, which helped us anticipate what may be needed in the policy. In particular, our IPV specialist shared the process that an IPV survivor goes through when seeking services at our hospital. This individual also acted as a patient/IPV survivor advocate during policy development, which helped us navigate potential barriers if we collaborated with an individual who experienced IPV (e.g., re-traumatization). The traumainformed expert on our team also helped us craft our policy so that it emphasized trauma-informed principles.

\section{Conclusions}

Creating a trauma-informed, IPV security policy is integral to bridging gaps in communication between hospital security and healthcare providers. An overarching policy provides a framework for practice and holds everyone accountable to the same standards. Engagement from security leaders is necessary for changing perceptions and attitudes towards trauma-informed approaches and creating a culture more supportive for survivors of IPV. Further, one must engage individuals across multiple disciplines during policy development to ensure that the policy is wellinformed and comprehensive. Interdisciplinary insight would also help formulate an evaluation plan at the time policy development begins, which is highly recommended to measure the fidelity of implementation and impact of the policy. Future directions and next steps include implementation and evaluation of our policy. Ultimately, improved integration of security officers within the healthcare team is crucial for supporting survivors of IPV and ensuring the safety and well-being of all individuals in the pediatric healthcare setting.

\section{Acknowledgments}

We thank Carol Murray, MSS, MLSP, and Mobola Owolabi, MPP, PMP, LSSGB for their work in developing of the policy; we thank Allison Curry, $\mathrm{PhD}, \mathrm{MPH}$ and Dominique Ruggieri, $\mathrm{PhD}$ for their general support and assistance in editing on the manuscript. We also thank Sherry Morgan, $\mathrm{PhD}$, MLS, RN for her general support in researching articles to inform policy development.

Funding: None.

\section{Footnote}

Data Sharing Statement: Available at http://dx.doi. org/10.21037/jhmhp-20-152

Conflicts of Interest: All authors have completed the ICMJE uniform disclosure form (available at http://dx.doi. org/10.21037/jhmhp-20-152). The authors have no conflicts of interest to declare.

Ethical Statement: The authors are accountable for all aspects of the work in ensuring that questions related to the accuracy or integrity of any part of the work are appropriately investigated and resolved.

Open Access Statement: This is an Open Access article distributed in accordance with the Creative Commons Attribution-NonCommercial-NoDerivs 4.0 International License (CC BY-NC-ND 4.0), which permits the noncommercial replication and distribution of the article with 
the strict proviso that no changes or edits are made and the original work is properly cited (including links to both the formal publication through the relevant DOI and the license). See: https://creativecommons.org/licenses/by-nc-nd/4.0/.

\section{References}

1. Jackson C, Newall M. Axios-Ipsos Coronavirus Index. New York: Axios-Ipsos, 2020. [cited 2020 Jun 30]. Available online: https://www.ipsos.com/en-us/news-polls/ axios-ipsos-coronavirus-index

2. Gracia E. Unreported cases of domestic violence against women: towards an epidemiology of social silence, tolerance, and inhibition. J Epidemiol Community Health 2004;58:536-7.

3. Huecker MR, King KC, Jordan GA, et al. Domestic violence. In: StatPearls. Treasure Island: StatPearls Publishing, 2021.

4. Campbell AM. An increasing risk of family violence during the Covid-19 pandemic: strengthening community collaborations to save lives. Forensic Sci Int Reports 2020;2:100089.

5. Bradbury-Jones C, Isham L. The pandemic paradox: the consequences of COVID-19 on domestic violence. J Clin Nurs 2020;29:2047-9.

6. Human Rights Watch. Police mishandling of sexual assault cases in the District of Columbia. New York: Human Rights Watch, 2013. [cited 2020 Jun 30]. Available online: https://www.hrw.org/report/2013/01/24/capitol-offense/ police-mishandling-sexual-assault-cases-district-columbia

7. Rancher C, Jouriles EN, McDonald R. Intimate partner violence, police involvement, and women's trauma symptoms. J Interpers Violence 2021;36:NP3510-23.

8. Hamberger LK, Rhodes K, Brown J. Screening and intervention for intimate partner violence in healthcare settings: creating sustainable system-level programs. J Womens Health (Larchmt) 2015;24:86-91.

9. Hamby S, Finkelhor D, Turner H, et al. Children's exposure to intimate partner violence and other family violence. Washington DC: Office of Justice Programs, 2011. [cited 2020 Mar 28]. Available online: https://www. ojp.gov/pdffiles1/ojjdp/232272.pdf

10. Corbin TJ, Purtle J, Rich LJ, et al. The prevalence of trauma and childhood adversity in an urban, hospitalbased violence intervention program. J Health Care Poor Underserved 2013;24:1021-30.

11. Marsac ML, Kassam-Adams N, Hildenbrand AK, et al. Implementing a trauma-informed approach in pediatric health care networks. JAMA Pediatr 2016;170:70-7.

12. Substance Abuse and Mental Health Services

Administration. SAMHSA's concept of trauma and guidance for a trauma-informed approach. Washington DC: U.S. Department of Health and Human Services, 2014. [cited 2020 Jun 30]. Available online: https://ncsacw. samhsa.gov/userfiles/files/SAMHSA_Trauma.pdf

13. Randell KA, Evans SE, O'Malley D, et al. Intimate partner violence programs in a children's hospital: comprehensive assessment utilizing a delphi instrument. Hosp Pediatr 2015;5:141-7.

14. Lynch TF, Horton R, Wynne MM. Taking the trauma out of security. J Healthc Prot Manage 2010;26:63-8.

15. Schoenfisch AL, Pompeii LA. Security personnel practices and policies in U.S. hospitals. Workplace Health Saf 2016;64:531-42.

16. Subramanian S, Green JS. The general approach and management of the patient who discloses a sexual assault. Mo Med 2015;112:211-7.

17. Ye J, Wang C, Xiao A, et al. Physical restraint in mental health nursing: a concept analysis. Int J Nurs Sci 2019;6:343-8.

18. Children's National Hospital. CHPC:CP:06 - Victims of family violence policy (intimate partner violence, child exposure to intimate partner violence and elder abuse). 2004.

19. Albert Einstein Medical Center. A01-002.2 - Intimate partner violence. 2019.

20. Children's Hospital of Philadelphia. Policy: intimate partner violence. 2017.

21. Children's Hospital of Philadelphia. 6-4 - Violence in the workplace policy. 2017.

22. Pennsylvania Coalition Against Domestic Violence. About abuse. Harrisburg: Pennsylvania Coalition Against Domestic Violence, 2020. [cited 2020 Jul 18]. Available online: https://www.pcadv.org/about-abuse/

23. Pennsylvania Coalition Against Domestic Violence. Types of abuse. Harrisburg: Pennsylvania Coalition Against Domestic Violence, 2020. [cited 2020 Jul 18]. Available online: https://www.pcadv.org/about-abuse/types-ofabuse/

24. National Domestic Violence Hotline. Abuse defined. Austin: National Domestic Violence Hotline, 2020. [cited 2020 Jul 18]. Available online: https://www.thehotline.org/ is-this-abuse/abuse-defined/

25. Pennsylvania Department of Human Services. Keep kids safe - who is a mandated reporter? Pennsylvania: Department of Human Services, 2020. [cited 2020 Jul 18]. Available online: http://www.keepkidssafe.pa.gov/about/ 
mandatedreporter/index.htm

26. Potterton J. Personal safety [security training presentation in PowerPoint]. Philadelphia: Children's Hospital of Philadelphia, 2019.

27. Potterton J. Family violence [security training presentation in PowerPoint]. Philadelphia: Children's Hospital of Philadelphia, 2020.

28. Potterton J. Verbal defense [security training presentation in PowerPoint]. Philadelphia: Children's Hospital of Philadelphia, 2020.

29. Protection from Abuse P.L.1240, No. 206, 1990 (Cwlth of PA).

30. Guedes A, Mikton C. Examining the intersections between child maltreatment and intimate partner violence. West J Emerg Med 2013;14:377-9.

31. Izaguirre A, Cater $\AA$. Child witnesses to intimate partner violence: their descriptions of talking to people about the violence. J Interpers Violence 2018;33:3711-31.

32. Swenson CC, Schaeffer CM, Schaeffer C. A multisystemic approach to the prevention and treatment of child abuse and neglect hhs public access. Int J Child Maltreat 2018;1:97-120.

33. The National Child Traumatic Stress Network. Secondary traumatic stress. Los Angeles: National Center for Child Traumatic Stress, 2020. [cited 2020 Jul 20]. Available online: https://www.nctsn.org/trauma-informed-care/ secondary-traumatic-stress

34. National Network to End Domestic Violence. Victim confidentiality considerations for domestic violence

doi: 10.21037/jhmhp-20-152

Cite this article as: Bautista AV, Obenschain KC, Potterton JT, Murray AL. Trauma-informed hospital security: a policy for intimate partner violence in a pediatric healthcare setting. J Hosp Manag Health Policy 202 1;5:39. and sexual assault programs when responding to rare or emergency situations. Harrisburg: National Network to End Domestic Violence, 2010. [cited 2020 Jul 18]. Available online: http://wscadv.org/wp-content/ uploads/2015/06/Confidentiality-and-EmergencySituations.pdf

35. U.S. Department of Health \& Human Services. Summary of the HIPAA security rule. Washington DC: U.S. Department of Health \& Human Services, 2012. [cited 2020 Jul 18]. Available online: https://www.hhs.gov/hipaa/ for-professionals/security/laws-regulations/index.html

36. Broaddus-Shea ET, Scott K, Reijnders $M$, et al. A review of the literature on good practice considerations for initial health system response to child and adolescent sexual abuse. Child Abuse Negl 2021;116:104225.

37. National Institute of Justice. National best practices for sexual assault kits: a multidisciplinary approach. Washington DC: National Institute of Justice, 2017. [cited 2020 Jul 18]. Available online: https://www.ojp.gov/ pdffiles1/nij/250384.pdf

38. Failure to report injuries by firearm or criminal act P.L.1350, No.162 2002 (Cwlth of PA).

39. Glasgow R, Boles S, Vogt T. What is RE-AIM. RE-AIM, 2020. [cited 2020 Jul 18]. Available online: http://www.reaim.org/about/what-is-re-aim/

40. Fischer F, Lange K, Klose K, et al. Barriers and strategies in guideline implementation-a scoping review. Healthcare (Basel) 2016;4:36. 